International Journal of Bifurcation and Chaos, Vol. 16, No. 9 (2006) 2559-2574

(c) World Scientific Publishing Company

\title{
ACTIVE AEROELASTIC CONTROL OF LIFTING SURFACES VIA JET REACTION LIMITER CONTROL
}

\author{
CHRISTINA RUBILLO* ${ }^{*}$ and PIERGIOVANNI MARZOCCA ${ }^{\dagger}$ \\ Department of Mechanical and Aeronautical Engineering, \\ Clarkson University, \\ Potsdam NY 13699-5725, USA \\ *rubillcm@clarkson.edu \\ ${ }^{\dagger}$ pmarzocc@clarkson.edu \\ ERIK BOLLT \\ Department of Mathematics and Computer Sciences \\ and Department of Physics, \\ Clarkson University, \\ Potsdam NY 13699-5815, USA
}

Received August 3, 2005; Revised September 9, 2005

\begin{abstract}
In this paper we present, the design and modeling of the novel nonlinear limiter control feedback control plant [Myneni et al., 1999; Corron et al., 2000; Corron \& Pethel, 2002], applied for the first time here in an aeroelastic system, and actuated as a jet reaction torquer control of a wing with potentially chaotic dynamics. This study will provide a better understanding of the nonlinear dynamics of the open/closed-loop aeroelasticity of flexible wings with either steady or unsteady aerodynamic loads. The limiter control can be applied to either the plunging or pitching characteristic of the wing or to both of them. We show that the control can effectively suppress Limit Cycle Oscillations (LCO) and chaos well beyond the nominal flutter speed. This could lead to a practical implementation of the control mechanism on actual and future generation aircraft wings via implementation of a combination of propulsive/jet type forces, micro surface effectors and fluidic devices. Analysis of this control produced favorable results in the suppression of LCO amplitude and increased flutter boundaries for plunging and pitching motion. The limiting control has asymptotically zero power, and is simply implemented, making it a feasible solution to the problem of the chaotic dynamics of the oscillating airfoil.
\end{abstract}

Keywords: Aeroelastic control; flutter; limiter control; Limit Cycle Oscillation (LCO).

\section{Introduction}

The tendency to reduce weight, increase structural flexibility and operating speed certainly increases the likelihood of the flutter occurrence within the aircraft operational envelope. Moreover, combat aircraft can experience, during their operational life, dramatic reductions of the flutter speed that can affect their survivability [Marzocca et al., 2001, 2002a, 2002b; Librescu et al., 2002, 2003a, 2003b; Qin et al., 2002]. The mission profile of the next generation of UAV will probably lead to a configuration requirement of an adaptable airframe to best meet the varying flight conditions. It is conceivable that the changes in geometry that occur would also incur

*Author for correspondence. 
aeroelastic instabilities, such as flutter, at points of transition during the mission.

Conventional methods of examining aeroelastic behavior have relied on a linear approximation of the governing equations of the flowfield and the structure. However, aerospace systems inherently contain structural and aerodynamic nonlinearities [Dowell, 1978] and it is well known that with these nonlinearities present, an aeroelastic system may exhibit a variety of responses that are typically associated with nonlinear regimes of response, including Limit Cycle Oscillation (LCO), flutter, and even chaotic vibrations [Dowell et al., 2003]. These nonlinearities result from unsteady aerodynamic sources, such as in transonic flow condition or at high angle of attack, large deflections, and partial loss of structural or control integrity. Aeroelastic nonlinearities have been identified in [Lee et al., 1999] and analyses, focusing on LCO behavior and flutter boundaries, have been performed on similar airfoils, as well as aeroelastic panels [Berggren, 2004; Shahrzad \& Mahzoon, 2002; Coller \& Chamara, 2004; Epureanu et al., 2004]. Previous work has shown that airfoils operating past the flutter boundary produce LCO that increase in amplitude with increased speed, limiting the safe flight boundary [Toumit \& Darracq, 2000].

The interest toward the development and implementation of active control technology was prompted by the new and sometimes contradictory requirements imposed on the design of the new generation of the flight vehicle that mandated increasing structural flexibilities, high maneuverability, and at the same time, the ability to operate safely in severe environmental conditions. In the last two decades, the advances of active control technology have rendered the applications of active flutter suppression and active vibrations control systems feasible [Marzocca et al., 2001; Mukhopadhyay, 2003]. A great deal of research activity devoted to the aeroelastic active control and flutter suppression of flight vehicles has been accomplished. The state-of-the-art advances in these areas are presented in [Horikawa \& Dowell, 1979; Vipperman et al., 1998]. The reader is also referred to a sequence of articles [Mukhopadhyay, 2000] where a number of recent contributions related to the active control of aircraft wing are discussed at length.

Early studies have shown that the flutter instability can be postponed and consequently the flight envelope can be expanded via implementation of a linear feedback control capability. However, the conversion of the catastrophic type of flutter boundary into a benign one requires the incorporation of a nonlinear feedback capability given a nonlinear aeroelastic system. In recent years, several active linear and nonlinear control capabilities have been implemented. Digital adaptive control of a linear aeroservoelastic model [Friedmann et al., 1997], $\mu$ method for robust aeroservoelastic stability analysis [Lind \& Brenner, 1999], gain scheduled controllers [Barker \& Balas, 2000], neural and adaptive control of transonic wind-tunnel model [Scott \& Pado, 2000; Guillot \& Friedmann, 2000] are only few of the latest developed active control methods. Linear control theory, feedback linearizing technique, and adaptive control strategies have been derived to account for the effect of nonlinear structural stiffness [Ko et al., 1997; Zhang \& Singh, 2001]. A model reference variable structure adaptive control system for plunge displacement and pitch angle control has been designed using bounds on uncertain functions [Zeng \& Singh, 1998]. This approach yields a high gain feedback discontinuous control system. In [Xing \& Singh, 2000], an adaptive design method for flutter suppression has been adopted while utilizing measurements of both the pitching and plunging variables.

This paper will present a simple and yet effective limiter jet reaction torquer control that would enable an increase in flutter speed, enhance the aeroelastic response, and suppress LCO and chaotic dynamics, preventing catastrophic failure. The nonlinear approach of lifting surfaces of aeronautical and space vehicles permits determination of the conditions under which undamped oscillations can occur at velocities below the flutter speed, and also of the conditions under which the flight speed can be exceeded beyond the flutter instability, without catastrophic failure, i.e. when a stable LCO takes place. The next generation of aircraft will not be mission specific, but will instead be able to adapt to many different situations and requirements, so the use of these controls to expand the safe flight boundary of an aircraft opens large opportunities in this direction. These facts emphasize the importance of at least two issues: (i) various nonlinear effects should be included in the aeroelastic analysis; (ii) implementing adequate control methodologies will enable one to expand the flight envelope by increasing the flutter speed, or to enhance the aeroelastic response by attenuating the excessive vibrations, but also to convert 
the unstable LCO into a stable one [Librescu \& Marzocca, 2005].

More insight into the wealth of the limiter control developed by Corron et al. [Corron et al., 2000; Corron \& Pethel, 2002; Myneni et al., 1999] and applied to this important aeroelastic problem is provided here. Limiter control uses small perturbations, in the form of a novel nonlinear feedback control plant, to neutralize system instabilities. While their techniques were primarily developed for electronic communications systems [Corron \& Pethel, 2002], this technique has been successfully applied to a chaotic mechanical system by Corron et al. [2000] and their chaotic driven pendulum experiment has proved its effectiveness. This pioneering work has shown that this dynamic limiting technique enables to selectively control unstable periodic orbits via minimal perturbations. Limiter control offers several advantages of system simplicity and feasibility, in particular simplicity for effective practical engineering, over previous parametric feedback methods more traditionally used in the field of controlling chaos, as reviewed and compared in [Bollt, 2003]. While other forms of nonlinear control of aeroelastic structural systems have previously been researched [Librescu \& Marzocca, 2005], the feasibility of limiter control as applied to a nonlinear airfoil, is explored in this paper. In Sec. 2, the mathematical model of the plunging/pitching airfoil will be introduced, as well as the limiter control. Section 3 outlines the results of the applied control on the airfoil in steady and unsteady flows, focusing on LCO suppression and flutter boundary extension. A brief summary of the conclusions can be found in Sec. 4 .

\section{Mathematical Model}

\subsection{Aeroelastic model}

A classical two degrees-of-freedom in pitch and plunge airfoil will be used to evaluate the limiter control. The schematic for this spring restrained, rigid wing model is shown in Fig. 1, where the springs represent the wing's structural bending and torsion stiffness. This model is referred to as a typical section, that can either represent a $2-\mathrm{D}$ wind tunnel model, or a section of a finite wing. The equations of motion are formulated from Lagrange's equations using potential and kinetic energies and generalized aerodynamic forces [Hodges \& Pierce, 2002]. The nonlinear aeroelastic governing equations are

$$
\begin{gathered}
\mu \ddot{h}+\mu \chi_{\alpha} \ddot{\alpha}+\zeta_{h} \dot{h}+\mu\left(\frac{\omega_{h}}{\omega_{\alpha}}\right)^{2} h=-\frac{Q_{h}}{\pi \rho b^{3} \omega_{\alpha}^{2}}+Q_{c, h} \\
\mu \chi_{\alpha} \ddot{h}+\mu r_{\alpha}^{2} \ddot{\alpha}+\zeta_{\alpha} \dot{\alpha}+\mu r_{\alpha}^{2} \alpha+\frac{\varepsilon}{\pi \rho b^{4} \omega_{\alpha}^{2}} \alpha^{3} \\
=\frac{Q_{\alpha}}{\pi \rho b^{4} \omega_{\alpha}^{2}}+Q_{c, \alpha}
\end{gathered}
$$

where $h$ and $\alpha$ are the plunge and pitching angle displacement, and $Q_{c, h}$ and $Q_{c, \alpha}$ represent the limiter control in plunge and pitch, respectively. In addition to the well-known structural terms, see [Marzocca 2002a, 2002b; Librescu et al., 2003a, 2003b], the aerodynamic lift and moment forces are represented by $Q_{h}, Q_{\alpha}$ respectively. The lift and moment are derived by summing the forces in each degree of freedom in equilibrium.

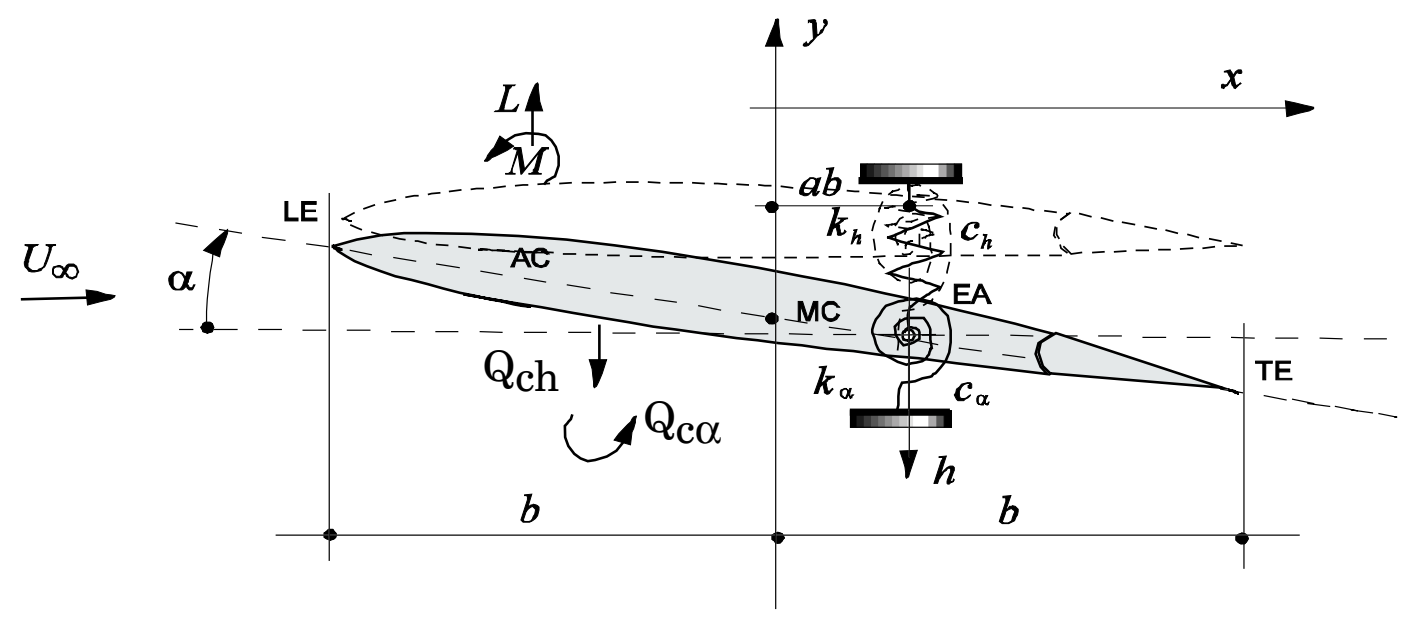

Fig. 1. 2-DOF pitching and plunging airfoil. 
A model with unsteady aerodynamics will also be considered. The lift and moment are expressed as [Fung, 1969]:

$$
\begin{aligned}
Q_{h}(\tau)= & -C_{L \alpha} b \rho U^{2} \int_{-\infty}^{\tau} \phi\left(\tau-\tau_{0}\right) \\
& \times\left[\dot{\alpha}+\frac{\ddot{h}}{b}+\left(\frac{1}{2}-a\right) \ddot{\alpha}\right] d \tau_{0} \\
& -\frac{1}{2} \rho C_{L \alpha} U^{2}(\ddot{h}-a b \ddot{\alpha})-\frac{1}{2} \rho C_{L \alpha} b U^{2} \dot{\alpha} \quad(3) \\
Q_{\alpha}(\tau)= & \left(\frac{1}{2}+a\right) C_{L \alpha} b^{2} \rho U^{2} \int_{-\infty}^{\tau} \phi\left(\tau-\tau_{0}\right) \\
& \times\left[\dot{\alpha}+\frac{\ddot{h}}{b}+\left(\frac{1}{2}-a\right) \ddot{\alpha}\right] d \tau_{0} \\
& +\frac{1}{2} a b \rho C_{L \alpha} U^{2}(\ddot{h}-a b \ddot{\alpha}) \\
& -\frac{1}{2}\left(\frac{1}{2}-a\right) \rho C_{L \alpha} b^{2} U^{2} \dot{\alpha}-\frac{1}{16} \rho C_{L \alpha} b^{2} U^{2} \ddot{\alpha}
\end{aligned}
$$

where $\phi(\tau)$ is the Wagner function and is approximated by [Jones, 1940]:

$$
\phi(\tau)=1-A_{1} e^{-b_{1} \tau}-A_{2} e^{-b_{2} \tau}
$$

where $A_{i}=(0.165 ; 0.335)$ and $b_{i}=(0.0455 ; 0.300)$. It should be remarked that in Eqs. (3) and (4), the coupling of plunging and pitch motions, referred to as aerodynamic coupling, appears explicitly. The unsteady aerodynamic lift and moment are split into circulatory and noncirculatory components. The integral terms appearing in Eqs. (3) and (4) correspond to the circulatory effect and are expressed, in the time domain, in terms of Wagner's function (also referred to as the heredity function). The remaining group of terms belong to the noncirculatory effects, and are referred to as added mass. These account for the inertia effects in the fluid, and are functions of the motion and the geometry of the airfoil section [Scanlan \& Rosenbaum, 1951]. In the next sections a steady and an unsteady model for the airfoil will be presented.

\subsection{Control methodology}

From the mathematical point of view, the nonlinear aeroelastic governing equations, Eqs. (1) and (2), will be coupled with a nonlinear feedback limiter control [Corron et al., 2000; Corron \& Pethel, 2002; Myneni et al., 1999]. Such control can stabilize desired orbits, and in this paper it is implemented toward suppressing LCO and chaotic motions [Bollt, 2003]. This technique has already been successfully applied to chaotic mechanical systems and experiments have proved its effectiveness. The limiter control technique [Corron et al., 2000; Corron \& Pethel, 2002] enables us to selectively control unstable periodic orbits via minimal perturbations. Furthermore, recent results have further extended the limiter control to allow for lowenergy control in electronic devices. The jet reaction torquer/morphing control can be mathematically described via a simple state dependent, but otherwise constant addition to the uncontrolled aeroelastic system, written in general multivariable form as [Bollt, 2003]:

$$
\dot{\mathbf{z}}=\mathbf{F}(\mathbf{z}, \mathbf{p})+\mathbf{G}(\mathbf{z}, t)
$$

where $\dot{\mathbf{z}}=\mathbf{F}(\mathbf{z}, \mathbf{p})$ represents the dynamics of the uncontrolled aeroelastic system. $\mathbf{G}(\mathbf{z}, t)$ represents the constant addition to the unperturbed dynamics and may be posed as combination of characteristics (indicator) functions, both spatially and temporally:

$$
\mathbf{G}(\mathbf{z}, t)=\sum_{n=0}^{\infty} \sum_{i=1}^{N} d_{n, i} \chi_{t_{n}}(t) \chi_{A_{i}}(\mathbf{z}) \mathbf{k}_{i} .
$$

For each fixed $i, d_{n, i}$ is a time-independent coefficient, and $\chi_{t_{n}}$ and $\chi_{A_{i}}$ are characteristic (indicator) functions of time and space, respectively. $\chi_{t_{n}}$ and $\chi_{A_{i}}$ are described as:

$$
\begin{gathered}
\chi_{t_{n}}(t)=\left\{\begin{array}{lc}
1 & \text { if } t_{n} \leq t \leq t_{n+1} \\
0 & \text { else }
\end{array}\right. \\
\chi_{A i}(t)=\left\{\begin{array}{lc}
1 & \text { if } t \in A_{i} \\
0 & \text { else }
\end{array}\right.
\end{gathered}
$$

where $A_{i}$ represents a region and the variable $\mathbf{k}_{i}$ is a constant vector addition, or direction the force should be applied, to the vectorfield whose influence tends to push the LCO in the general direction of $\mathbf{k}_{i}$. This should be chosen appropriately to suppress the LCO and chaotic motions. This concept is demonstrated in Fig. 2. A qualitative explanation of this control is that when the dynamics of the system enter the region, $A_{i}$, for example when the airfoil reaches a predefined deflection limit, the control is applied to push it back to that limit. For the form of the limiter control mechanism, shown below for both plunging and pitching, we choose

$$
\begin{aligned}
& Q_{c, h}=g_{h} H\left(h-\delta_{h}\right) \\
& Q_{c, \alpha}=g_{\alpha} H\left(\alpha-\delta_{\alpha}\right)
\end{aligned}
$$




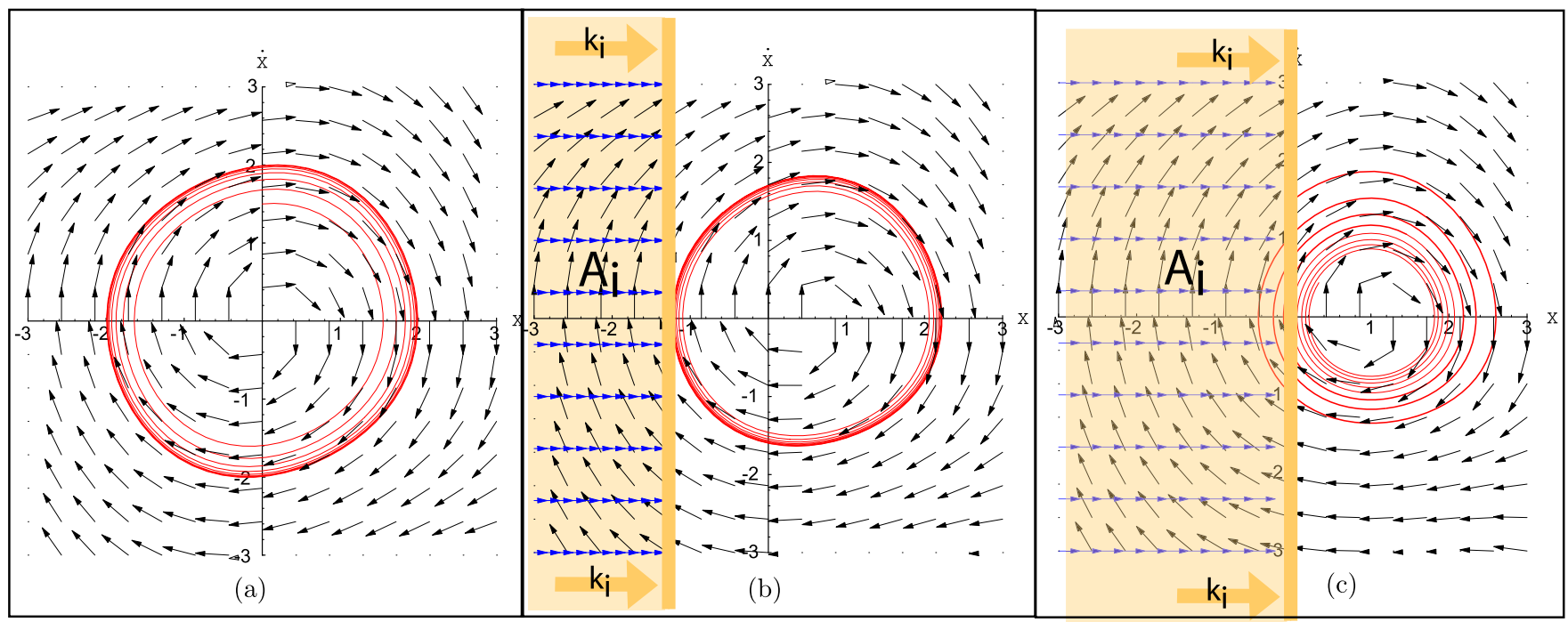

Fig. 2. Position versus velocity plot of a simple limit cycle (a) without control, (b) with a control value, $\mathbf{k}_{i}$, that decreases the LCO amplitude, (c) with a control value that completely damps-out the system. The shaded area represents the direction of the control value $\mathbf{k}_{i}$ and the magnitude of $\mathbf{G}(\mathbf{z}, t)$. $\mathrm{A}_{i}$ represents the region for which the control is applied.

This form of the control is applied in Eqs. (12) and (12). In other words the characteristic functions in Eq. (7) are simplified here as the Heavyside function, $H$. The control gain, or direction of the pushing force, is represented by $g_{h}$ and $g_{\alpha}$, while the control perturbation is given as $\delta_{h}$ and $\delta_{\alpha}$. For this model the control gain can be either positive or negative since the force addition can come from either direction. When applied to the corresponding plunge or pitch governing equation this control produces the desired vector addition. Some explanatory cases will be presented in the next section for the 2-D wing model first controlling the plunging characteristics, followed by the pitching for both steady and unsteady models. Both models governing equations were numerically solved using Mathematica, which utilizes a combination of a nonstiff Adams method and a stiff Gear method. The Adams scheme is implicit and the Gear method is based on a backward difference scheme [Wolfram, 1999].

\section{Results and Discussion}

\subsection{Steady model}

A linear model of the airfoil in steady flow will be analyzed first. The following parameters were used: $\mu=12.8, b=0.118, \zeta=0.2, \omega_{h}=34.6, \omega_{\alpha}=88$, $r_{\alpha}^{2}=0.3, \varepsilon=20$ and $\chi_{\alpha}=0.15$, from [Shahrzad \& Mahzoon, 2002]. For the steady flow the governing equations become:

$$
\begin{aligned}
12.8 \ddot{h} & +1.92 \ddot{\alpha}+0.2 \dot{h}+1.97 h \\
& =-\frac{1}{\pi}\left(\frac{U}{b \omega_{\alpha}}\right)^{2}(5.568 \alpha+0.0942)+Q_{C, h} \\
1.92 \ddot{h} & +3.84 \ddot{\alpha}+1.2 \dot{\alpha}+3.84 \alpha+4.24 \alpha^{3} \\
& =\frac{1}{\pi}\left(\frac{U}{b \omega_{\alpha}}\right)^{2}(1.4845 \alpha+0.0084)+Q_{C, \alpha}
\end{aligned}
$$

Figure 3 shows the phase portraits for the steady model before and after flutter speed. For the steady model the flutter speed was found to be $17.22 \mathrm{~m} / \mathrm{s}$, which is consistent with the value obtained by [Shahrzad \& Mahzoon, 2002]. These phase portraits show that after flutter speed a limit cycle is reached, with amplitude of 0.17 radians for pitching.

Before we applied any control, we analyzed the effect of the nonlinear stiffness factor, $\varepsilon$, on LCO amplitude. As shown in Fig. 4, increasing this stiffness factor significantly decreases the LCO amplitude, while the flutter speed remains constant. As a limiting case, $\varepsilon=0$, a straight vertical line at the flutter speed will be obtained.

Analysis of the steady model's flutter speed behavior, under plunge control, shows no significant change. As the control gain, $g_{h}$, and the control perturbation, $\delta_{h}$, vary, the flutter speed remains almost constant at its uncontrolled value. This is in agreement with the findings reported in [Librescu \& Marzocca, 2005]. Under pitch control, a considerable change in flutter speed is observed. 


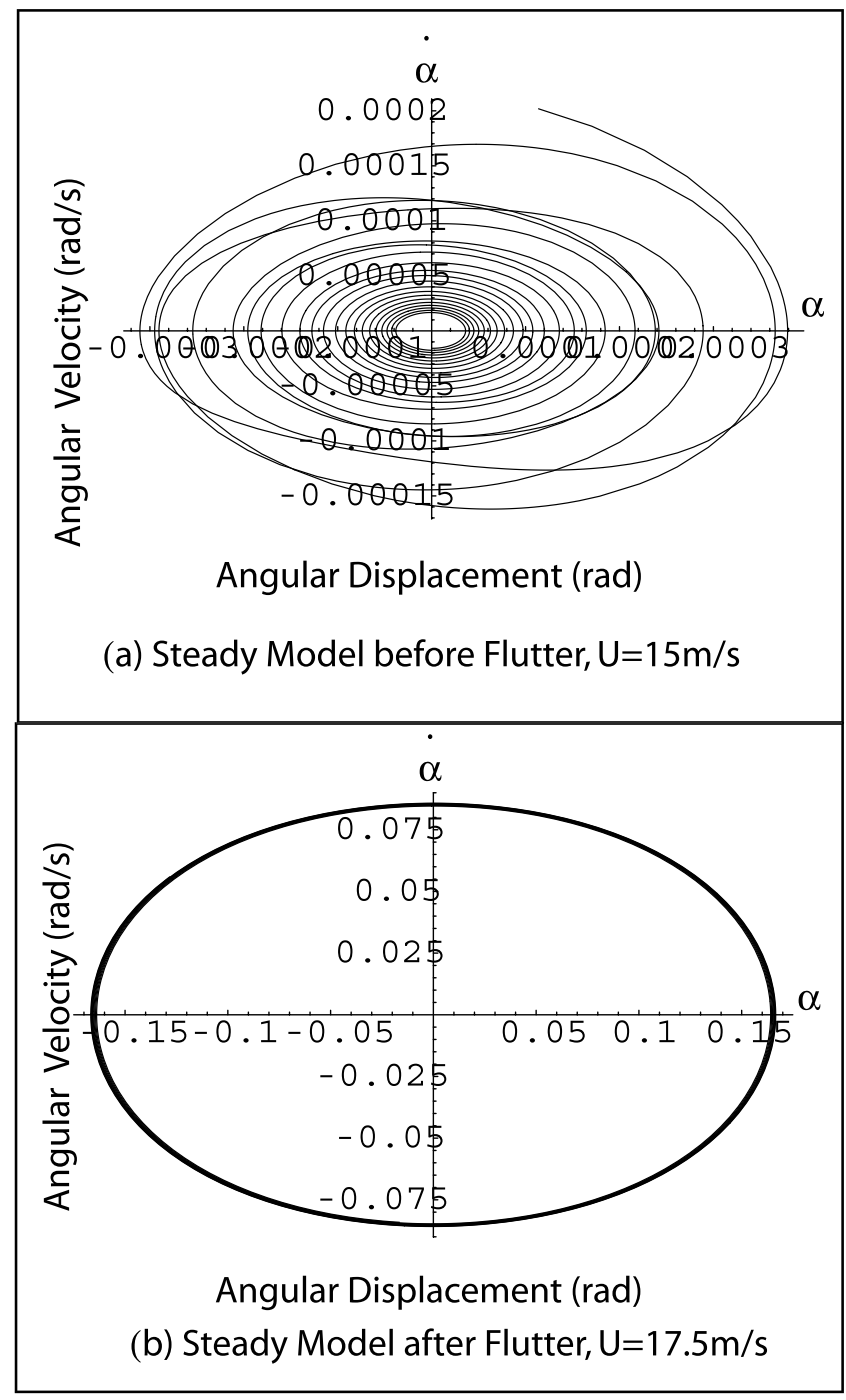

Fig. 3. Phase plots depicting the LCO of the airfoil under steady flow conditions: (a) before flutter, converging to zero, $U=15 \mathrm{~m} / \mathrm{s}$, (b) after flutter with LCO behavior, $U=$ $17.5 \mathrm{~m} / \mathrm{s}$.

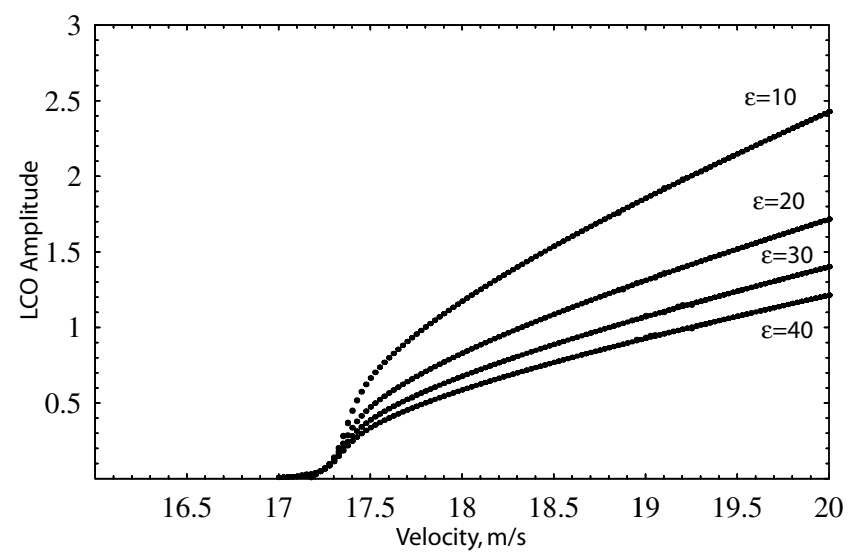

Fig. 4. Velocity versus LCO amplitude for various nonlinear stiffness factors, $\varepsilon$.
The potential of pitch control in the steady model is highlighted in Fig. 5. The amplitude of the LCO versus the free stream velocity for a plunging/pitching airfoil with a freeplay structural nonlinearity in pitch, subjected to an incompressible flow is presented. When this airfoil's flutter speed $U_{F}$ is exceeded, the airfoil experiences a stable LCO and a chaotic behavior. This analysis shows that the limiter control is capable of suppressing LCO and also the chaotic dynamics of such an airfoil, even well beyond the flutter speed. Notice the Hopf bifurcation that occurs at flutter speed, $U_{F}$. Before this point the system is converging, however above flutter speed a stable LCO appears. At approximately $U=25$, the system becomes chaotic as shown in the magnified region. The plots shown below the curve represent the system with the applied control. The stable limit cycle is now converging and the chaotic region has also been suppressed. Subjecting the steady airfoil model to pitch control at a velocity close to flutter speed can significantly damp out the system. In Fig. 6, the pitching time histories are shown for a velocity of $17.3 \mathrm{~m} / \mathrm{s}$ with and without control. The uncontrolled airfoil slowly approaches a limit cycle. The time history displays the amplitude of vibration shortly before reaching the LCO (approximately at $\tau=1800$ ) and including the LCO. By applying pitch control $g_{\alpha}=-1$, the oscillations stop before $\tau=350$ (2.3 seconds).

\subsubsection{Flutter analysis}

The effect of pitching control gain, $g_{\alpha}$, and control perturbation, $\delta_{\alpha}$, on flutter speed is shown in Fig. 7. This effect is very complex, but there exist combinations of $g_{\alpha}$ and $\delta_{\alpha}$, for which the control is most effective in extending the flutter boundary. One can see that, for control gains between -0.1 and 0.1 , an increase in the negative direction at a very small control perturbation value extends the flutter boundary. As the magnitude of the control gain increases in the positive direction, the opposite behavior is observed. Focusing on the larger range, it is apparent that for gains with an amplitude larger than 0.1 , the flutter speed will gradually drift to the uncontrolled flutter speed and then continually decline for positive gains and steadily decline for negative gains. In the negative direction, any gain with a magnitude larger than 0.1 decreases the flutter speed, while gains between 0 and -0.1 produce a useful increase in the flutter speed. 


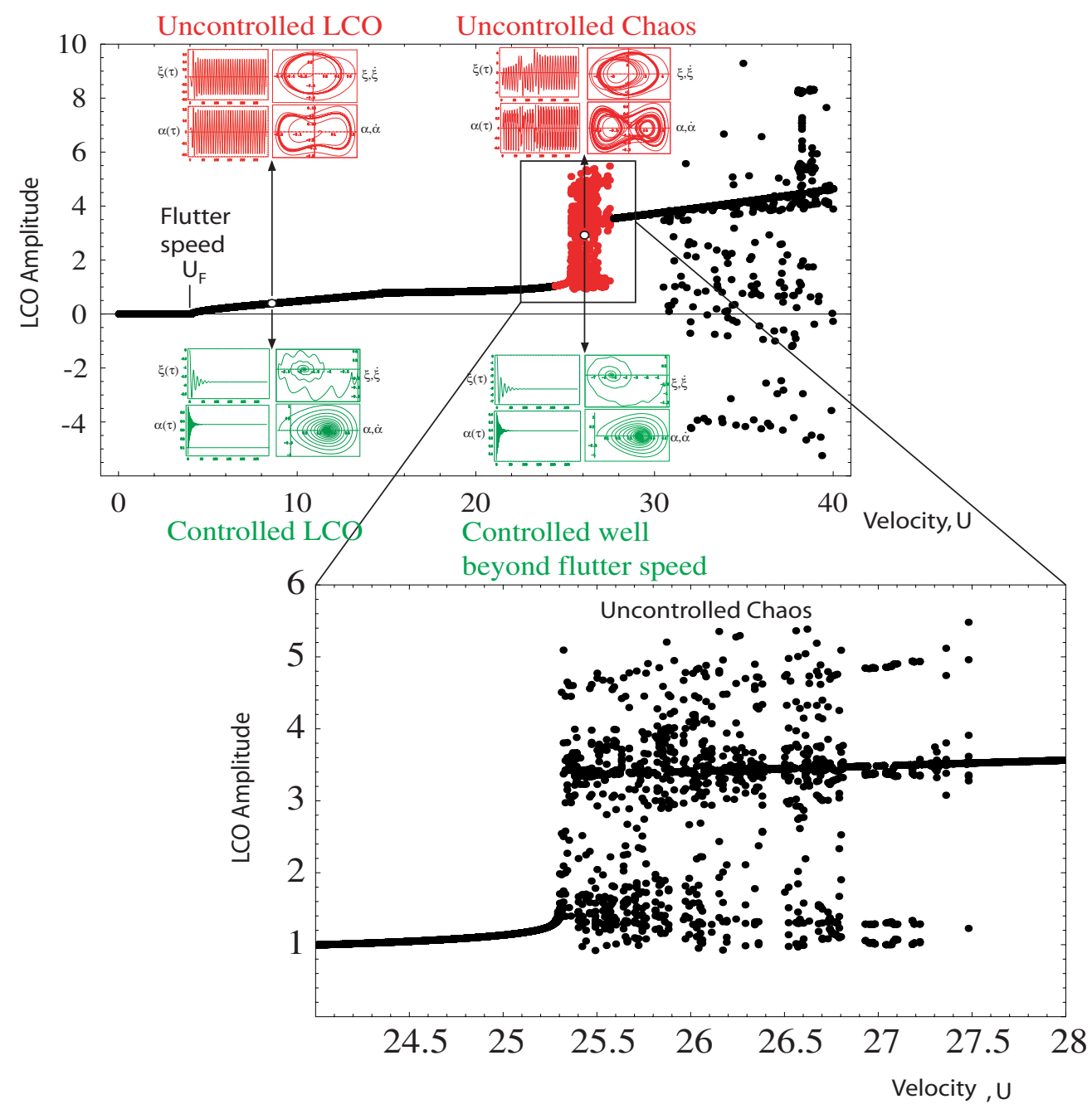

Fig. 5. LCO amplitude versus free stream velocity for a 2-DOF system. $U_{F}$ represents the flutter speed, after which a stable LCO appears. After approximately $U=25$, a chaotic region exists. The phase portraits and time histories above and below the curve represent the uncontrolled and controlled systems, respectively.

Figure 7 also shows the behavior of the flutter speed as we vary the control perturbation, $\delta_{\alpha}$. Focusing primarily on the left-hand side of the graph, one can observe that the lowest value of $\delta_{\alpha}$, produces the desired result. The flutter speed is greatly increased in the smaller control gain magnitude range. For larger perturbations the flutter speed is not improved, but decreased, further limiting the safe flight boundary. Considering the $\delta_{\alpha}$ value as delay, these results are consistent with [Librescu \& Marzocca, 2005]. It was shown that small time delay in the control could be advantageous, whereas large time delay could destabilize the system. Perturbations larger than 0.05 produce flutter speed that is equivalent to the value of the uncontrolled boundary. Since $\delta_{\alpha}$ is a perturbation to the angle of attack, its value should remain small and on the order of magnitude of the existing angle.
From a design perspective, this analysis could guide engineers toward selecting appropriate ranges of $g_{\alpha}$ and $\delta_{\alpha}$, for maximum performance.

\subsubsection{LCO amplitude analysis}

The LCO amplitude of the steady model was also examined under both plunging and pitching control. Although the flutter boundary was not extended under plunge control, the LCO amplitude is slightly suppressed at lower speeds. From results not displayed in this paper it can be concluded that for higher control gains the region before the flutter speed is unstable. This is consistent with [Librescu \& Marzocca, 2005], where it was demonstrated that an optimal gain value exists. Plunging control gains should be kept small for the best result in both flutter and LCO behavior. Under pitch control, the 


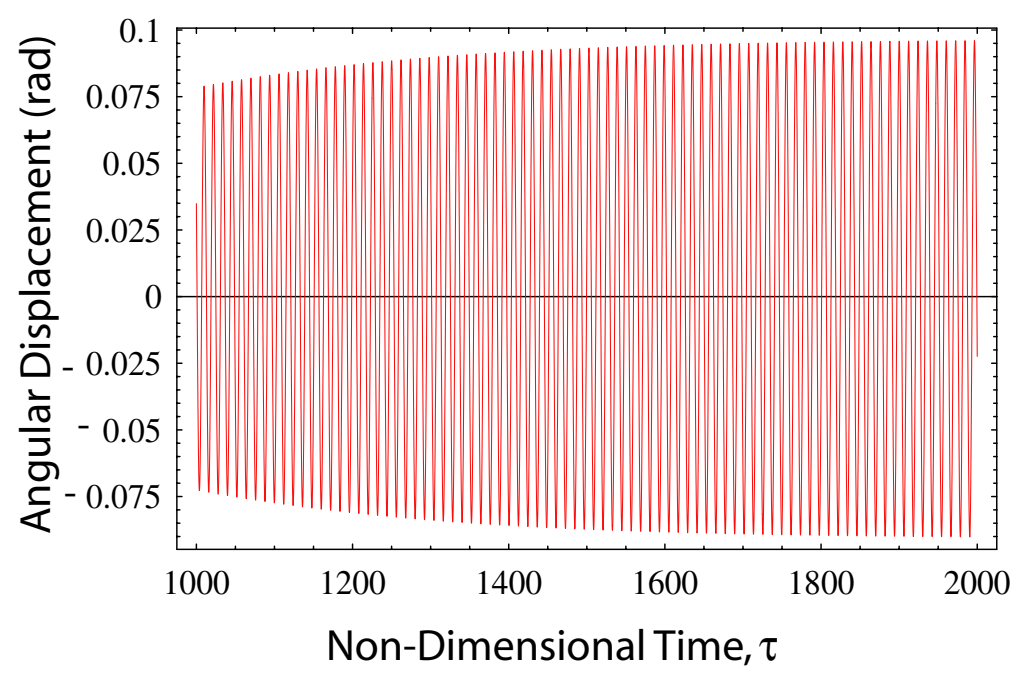

(a) No Pitch Control

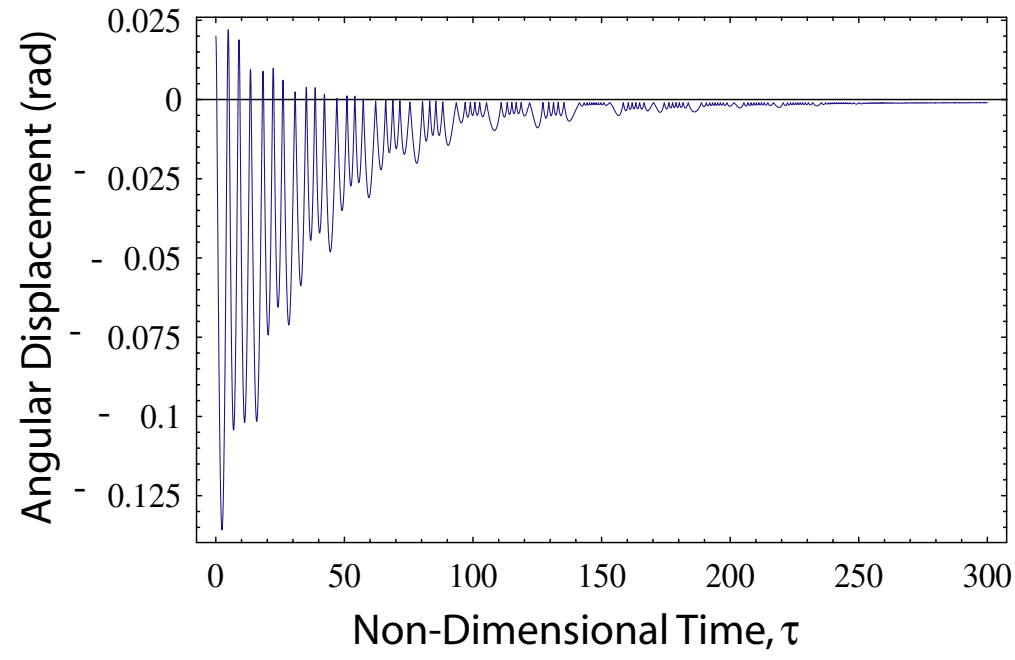

(b) Pitch Control Gain, $g_{\alpha}=-1$

Fig. 6. Time histories of airfoil at $U=17.3 \mathrm{~m} / \mathrm{s}$ (a) without control, (b) with pitching control gain, $g_{\alpha}=-1, \delta_{\alpha}=0.001$, showing that pitch control can damp the oscillating airfoil.

LCO amplitude is suppressed with larger control gains in the negative direction. The amplification of the LCO with larger control gains is shown in Fig. 8. After $g_{\alpha}=-0.25$ the system becomes chaotic. The pitching control perturbation, $\delta_{\alpha}$, also contributes to LCO suppression. Figure 9 shows the LCO amplitude as $\delta_{\alpha}$ is changed for a positive and negative control gain. Comparing this to the uncontrolled LCO, one can see that when applying a negative control gain an increase in the control perturbation in the positive direction slightly suppresses the LCO. Increasing the amplitude of perturbation in the negative direction slightly amplifies the LCO.
When applying a positive control gain, the opposite occurs.

\subsubsection{Control power analysis}

Another pertinent aspect of the limiter control presented in this paper is the power needed to maintain a stable LCO once control is applied. After control is applied the transient loops are "pushed" toward a limit cycle. The power and time plot shows how much power is required to push each transient loop to the LCO. Figure 10 highlights the area that was observed. The points that are 


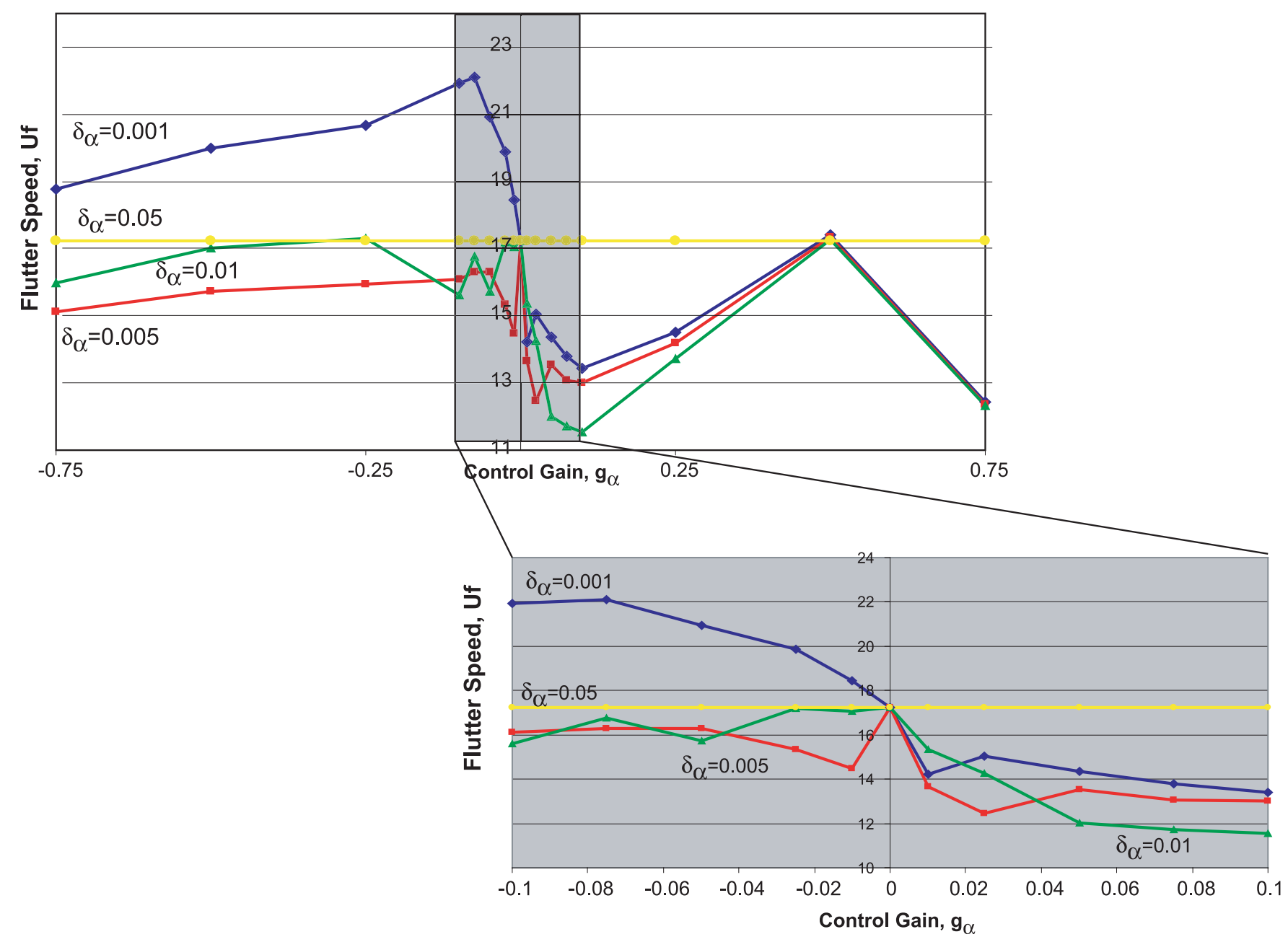

Fig. 7. Control gain, $g_{\alpha}$, versus flutter speed for an airfoil under steady conditions with pitch control and various control perturbations.

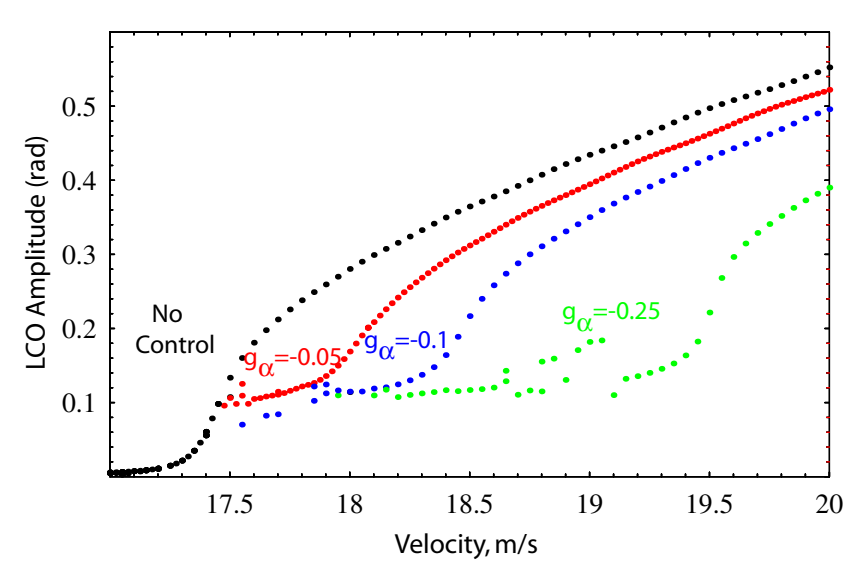

Fig. 8. Free stream velocity versus LCO amplitude under pitch control. Various values of the control gain are shown, $\delta_{\alpha}=0.001$.

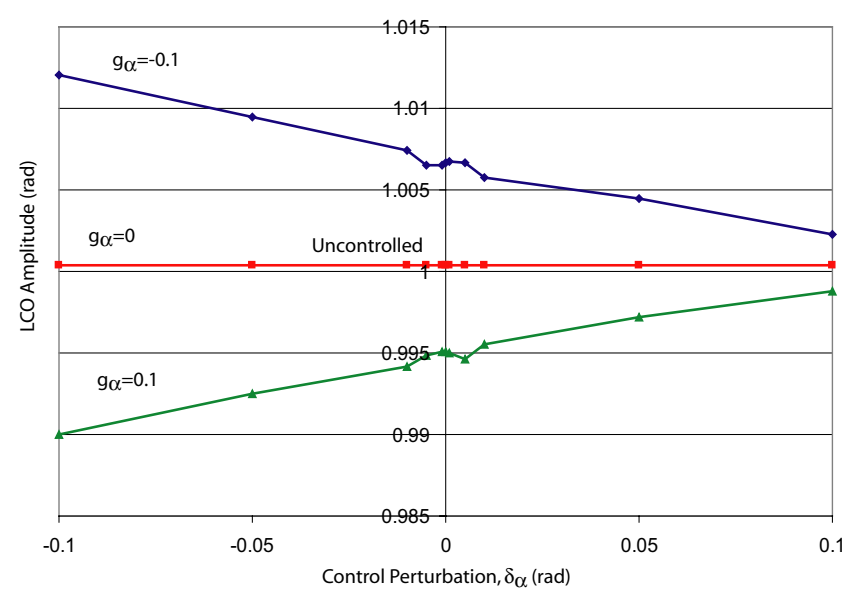

Fig. 9. Pitch control perturbations, $\delta_{\alpha}$, versus LCO Amplitude for various control gains, $g_{\alpha}$. 


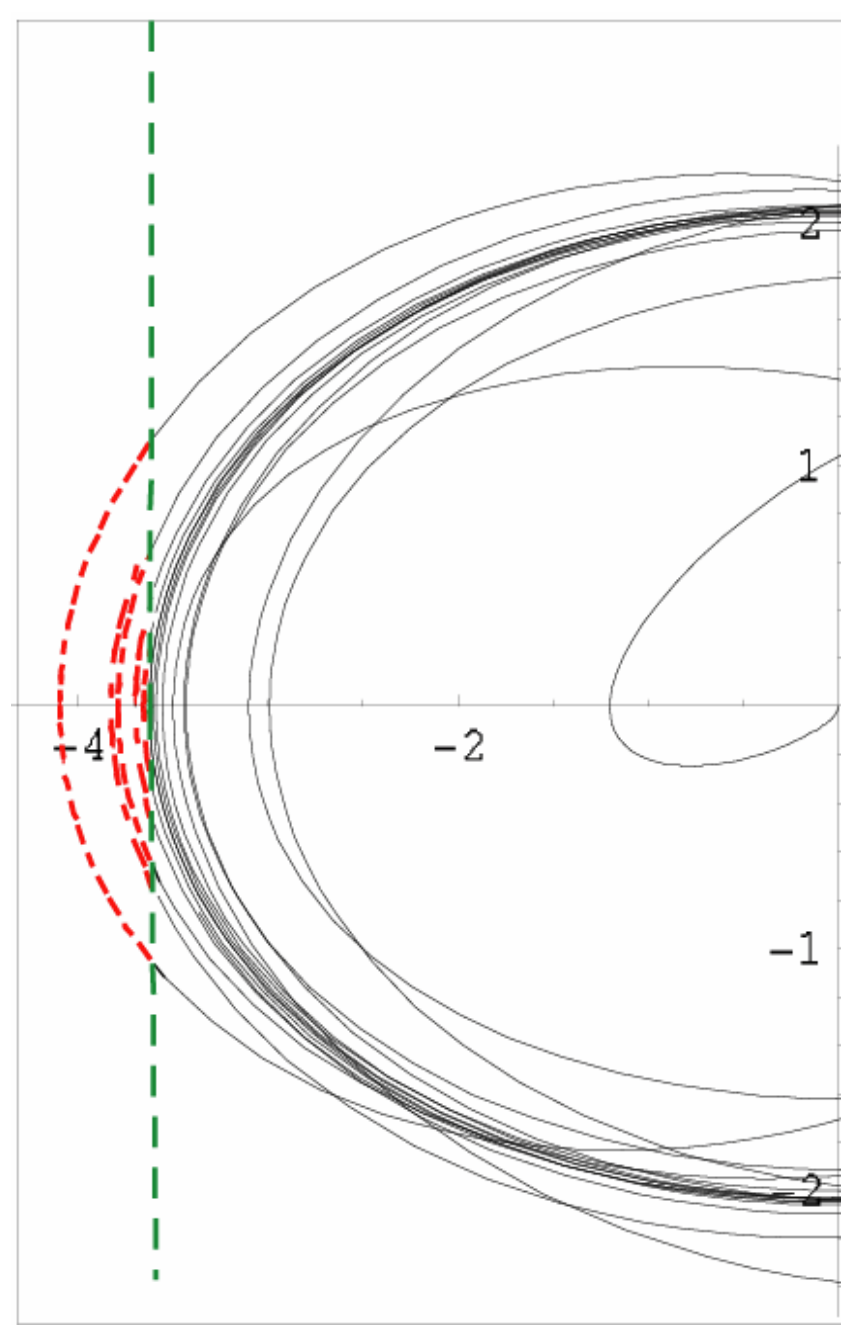

Fig. 10. Observed Region for limiter control power analysis. shown past the vertical dotted line are those where the position is less than the limit cycle. The vertical line represents the time and amplitude of the LCO. Figures 11 and 12 display a representative power plot for plunging and pitching gains, respectively. The control gain and perturbation are small and the free stream velocity is $25 \mathrm{~m} / \mathrm{s}$. Maintaining the LCO in plunge control, requires several larger power outputs with less frequency, while the pitch control needs smaller amplitude power outputs at a higher frequency. Both plots show that a relatively small amount of power is needed to maintain the smaller limit cycle. Notice that the required power is asymptotically decreasing toward zero as the controlled LCO is reached. Eventually the system would remain at the controlled LCO with little to no power applied.

\subsubsection{Chaos analysis}

An analysis of the airfoil in steady flow at high speeds shows that a state of chaos is reached. A phase portrait and time history of the system under chaotic conditions is shown in Fig. 13. Looking at the phase portrait it is difficult to understand or predict the airfoil's behavior. In order to better define what occurs a Poincaré plot was formulated. The maximum amplitude was plotted versus velocity, also shown in Fig. 13. In this plot it is apparent that four maximums exist. Although the system is chaotic the Poincaré plot shows a pattern.

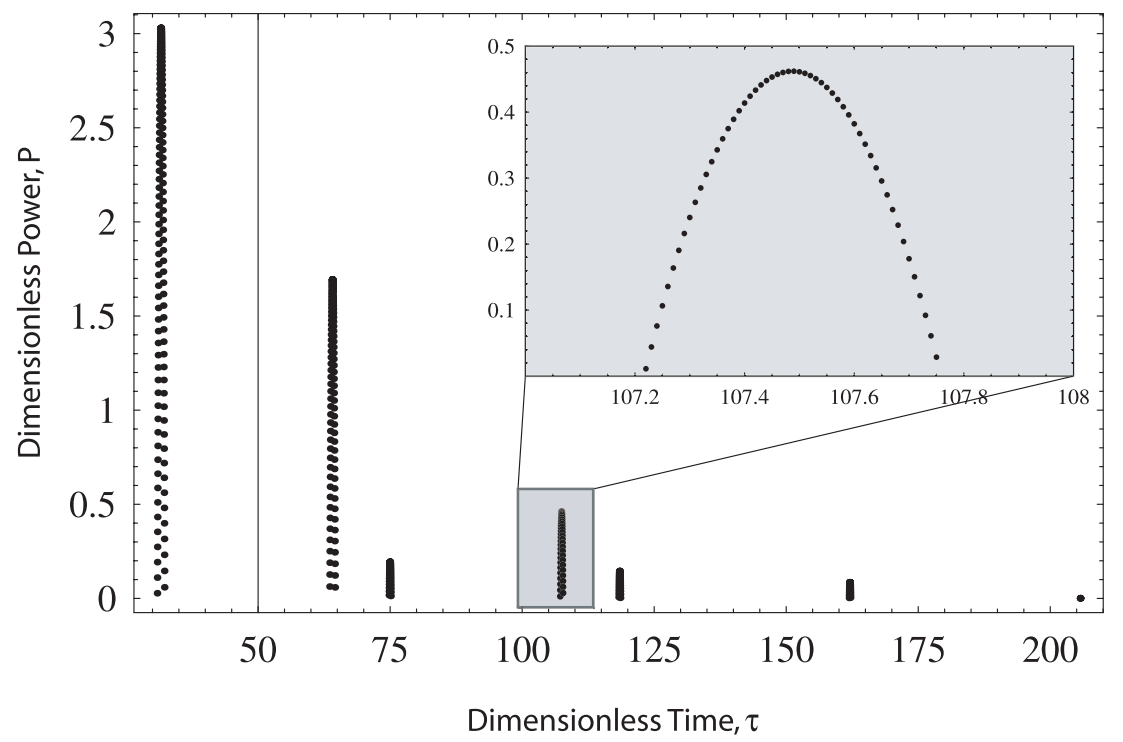

Fig. 11. Power versus time for steady model with plunge control showing that the power needed to control the LCO is asymptotically zero. 


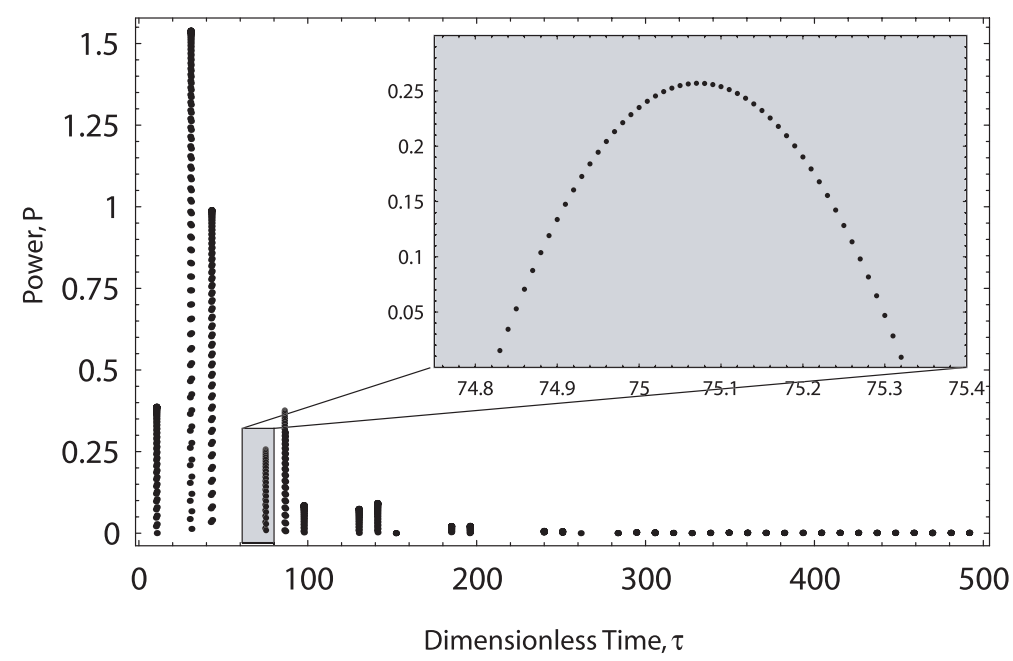

Fig. 12. Power versus time for steady model with pitch control showing that the power needed to control the LCO is asymptotically zero.
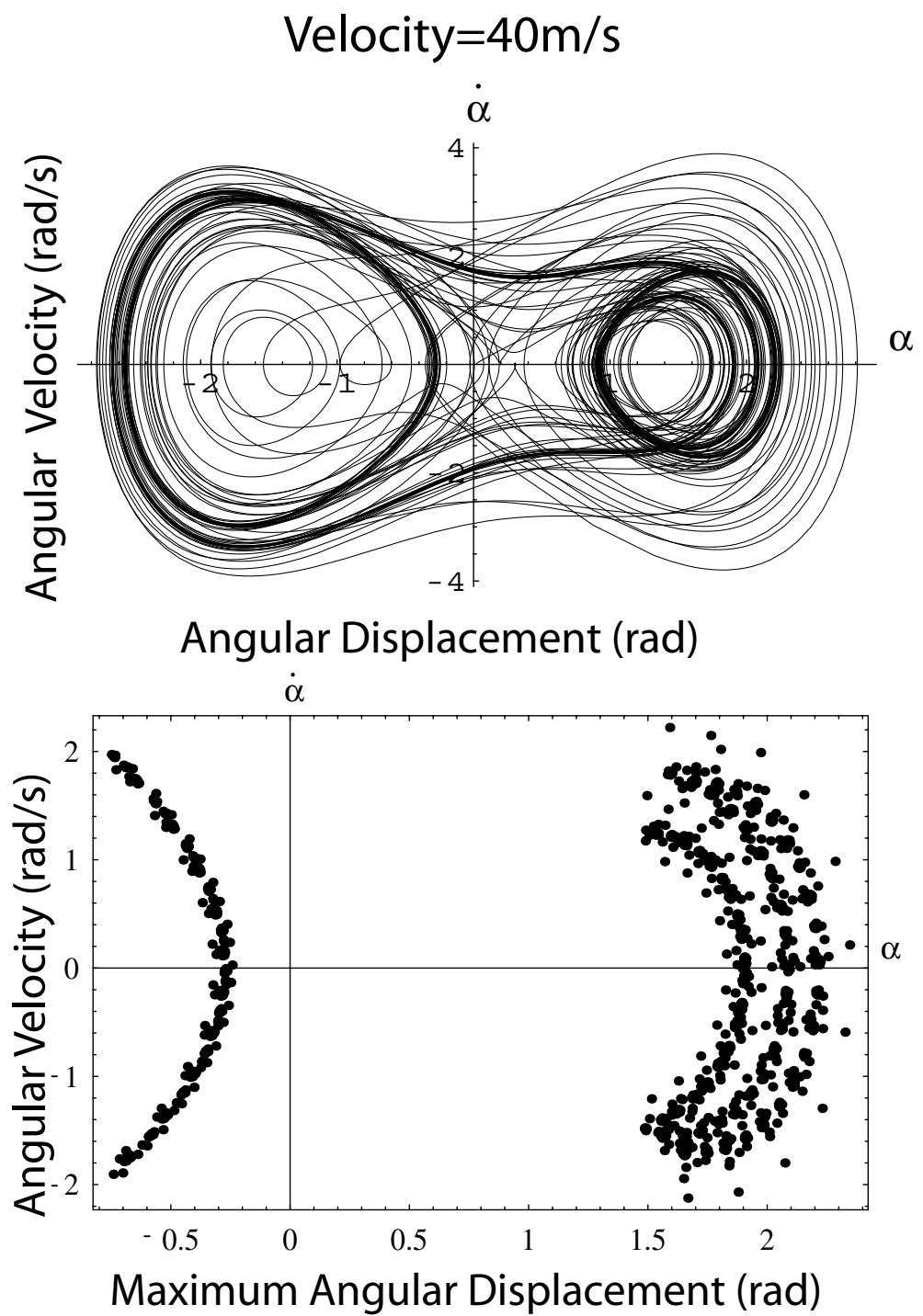

Fig. 13. Phase portrait of steady model exhibiting chaotic behavior at $40 \mathrm{~m} / \mathrm{s}$ and Poincaré plot of maximum angular displacement versus velocity showing four maximums. 
To further clarify the behavior, a Poincaré plot of velocity and displacement was created, where the displacement is only plotted when it is in phase with $2 \pi$. This creates a "strobed" phase portrait, Fig. 14. By strobing the phase portrait we see that, although the system is chaotic, the points fall in almost the same place each time. These plots may prove useful in understanding when to apply the limiter control in a chaotic system.

\subsection{Unsteady model}

A preliminary analysis of an airfoil under unsteady flow conditions was performed. The following parameters were used: $\mu=13.8, b=0.118, \zeta=0.2$,

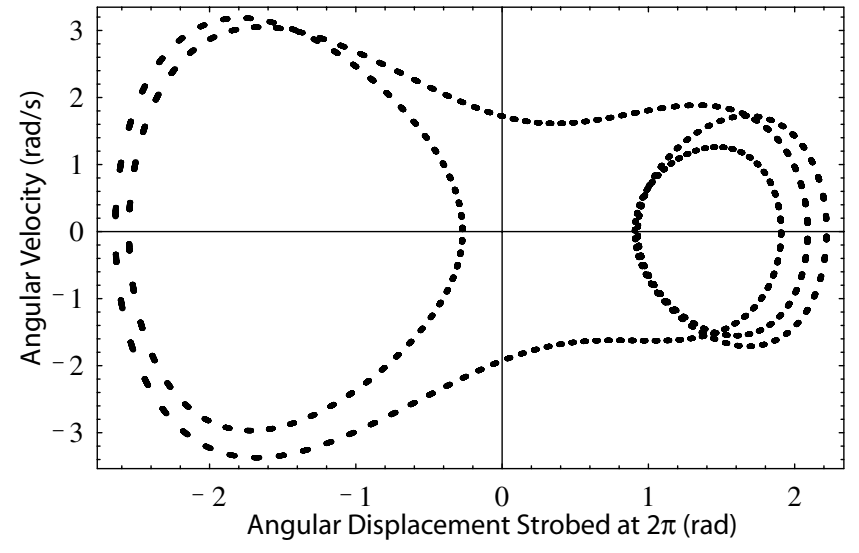

Fig. 14. Strobed Poincaré plot of maximum angular displacement versus velocity when displacement is in phase with $2 \pi$.

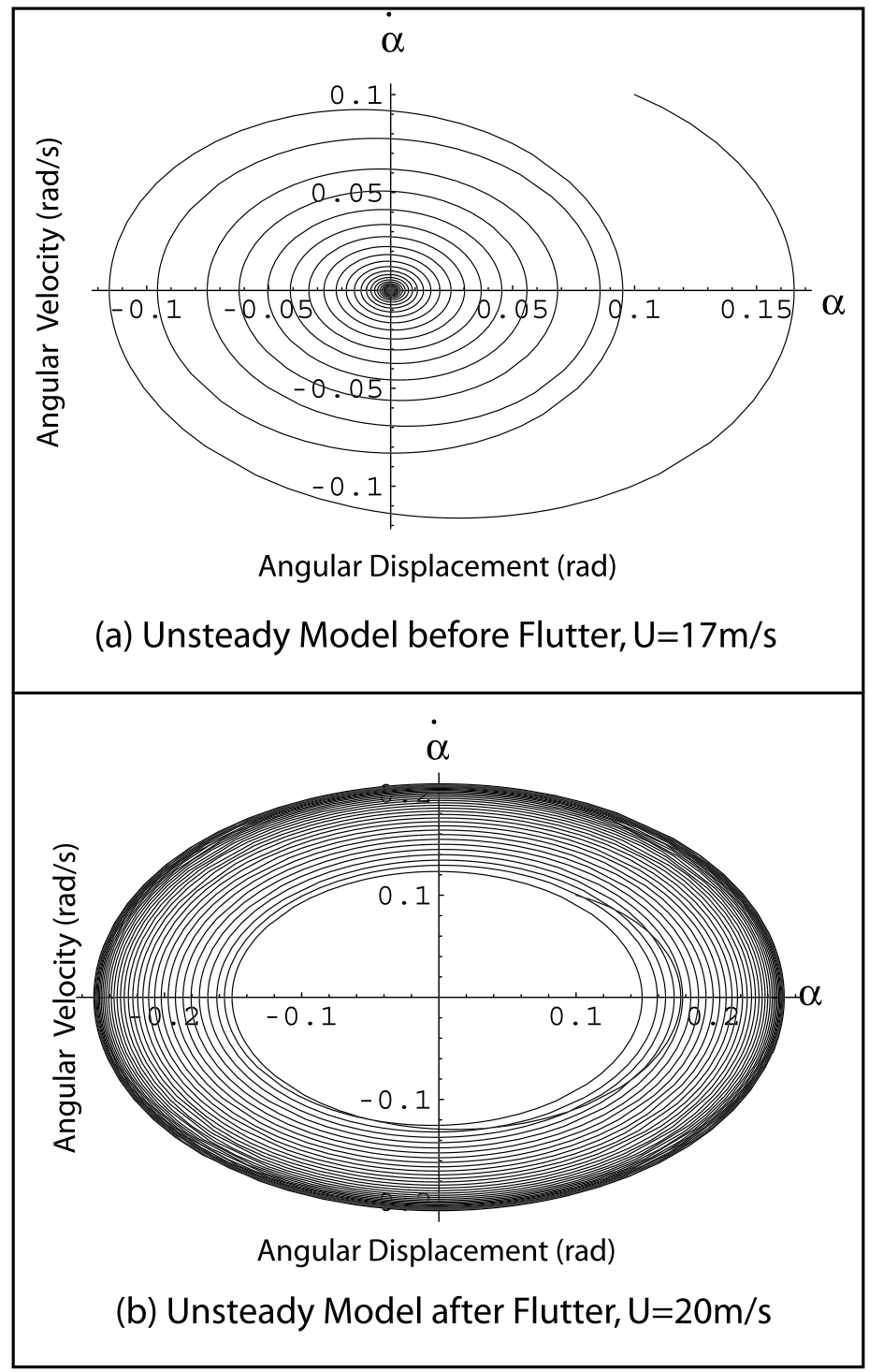

Fig. 15. Phase plots depicting the LCO of the airfoil under unsteady flow conditions: (a) before flutter, converging to zero, $U=17 \mathrm{~m} / \mathrm{s}$, (b) after flutter, exhibiting an LCO, $U=20 / \mathrm{s}$. 


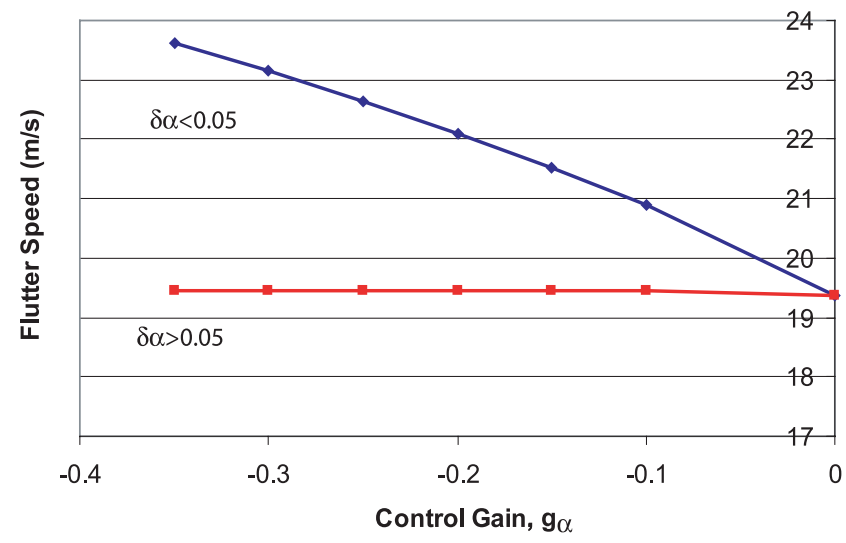

Fig. 16. Pitching control gain versus flutter speed for unsteady model.

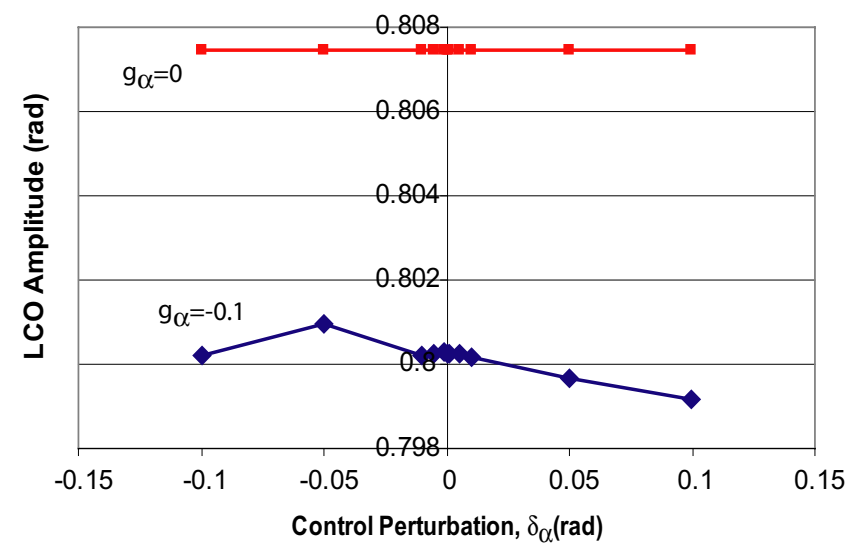

Fig. 17. Pitching control gain versus flutter speed for unsteady model.

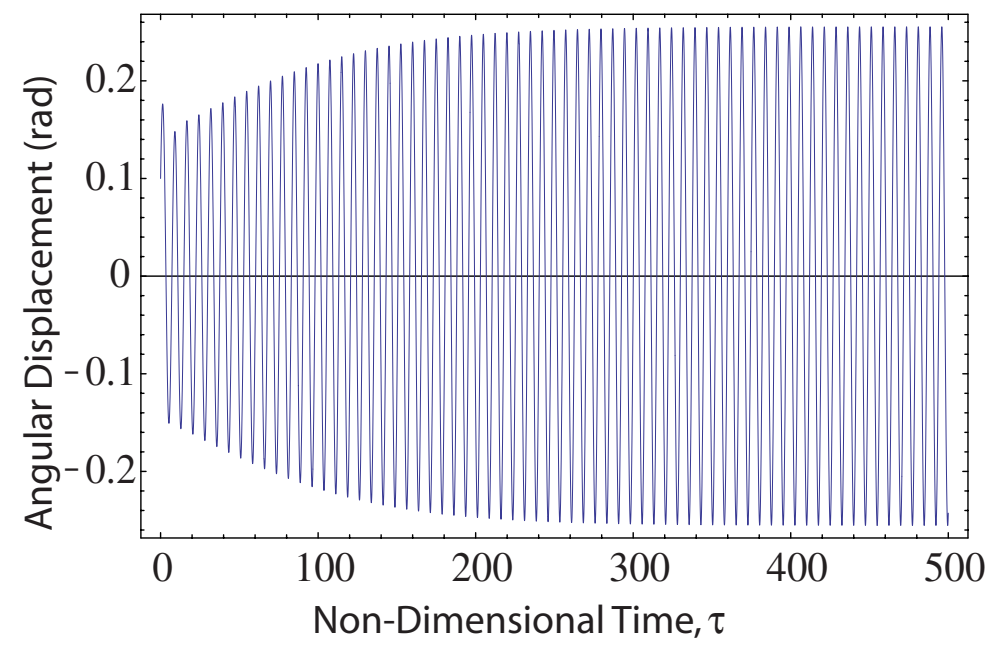

(a) No Pitch Control

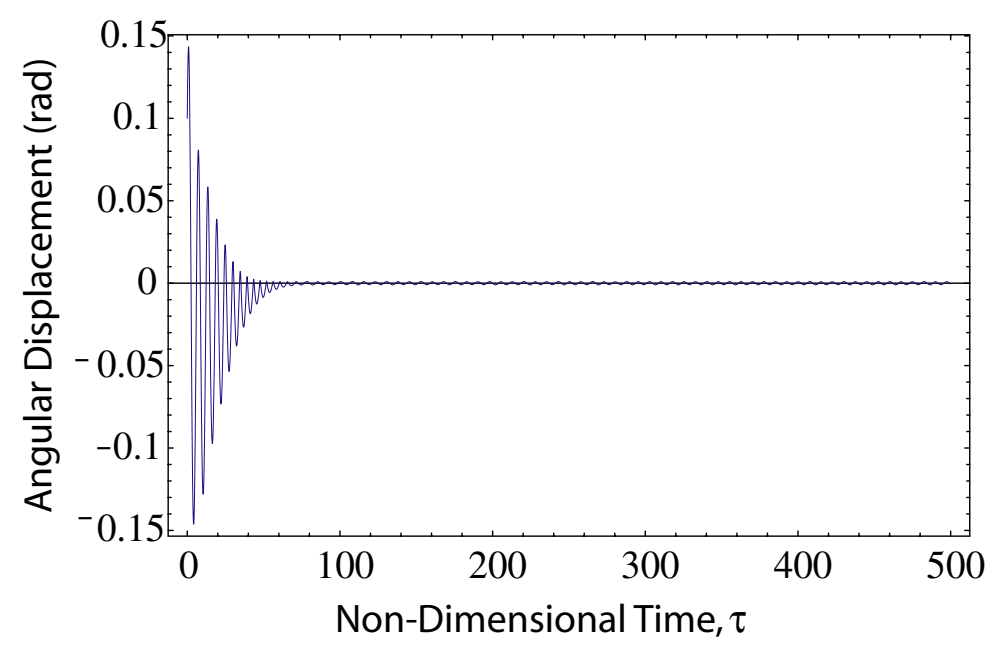

(b) Pitch Control Gain, $g_{\alpha}=-0.25$

Fig. 18. Time histories of unsteady airfoil at $U=20 \mathrm{~m} / \mathrm{s}$ (a) without control, (b) with pitching control gain, $g_{\alpha}=-0.25$, $\delta_{\alpha}=0.001$, showing that the LCO of an unsteady airfoil can be suppressed with this control. 
$\omega_{h}=34.6, \omega_{\alpha}=88, r_{\alpha}^{2}=0.3, \varepsilon=20$ and $\chi_{\alpha}=0.15$. The motion of this airfoil in plunging and pitching can be expressed as [Shahrzad \& Mahzoon, 2002]:

$$
\begin{aligned}
& 13.8 \ddot{h}+ 2.33 \ddot{\alpha}+(0.2+0.1926 U) \dot{h}+1.9787 h \\
&+ 0.2715 U \dot{\alpha}+0.01854 U^{2} \alpha=-Q_{C, h} \\
& 2.33 \ddot{h}+4.1331 \ddot{\alpha}+(0.2+0.0718 U) \dot{\alpha} \\
&+ \\
&+\left(3.84-0.001669 U^{2}\right) \alpha+4.24 \alpha^{3} \\
&-0.01733 U \dot{h}=Q_{C, \alpha}
\end{aligned}
$$

Figure 15 shows the phase portraits for the unsteady model before and after flutter speed. For the unsteady model the flutter speed was found to be $19.38 \mathrm{~m} / \mathrm{s}$, this is consistent with the results obtained by [Shahrzad \& Mahzoon, 2002]. These phase portraits show that after flutter speed a limit cycle is reached, with amplitude of 0.34 radians for pitching.

Applying limiter control to the plunging movements was found to have no positive effect on the flutter boundary. As the magnitude of the control gain increases in the positive direction, the flutter speed remains constant at the uncontrolled value. Increasing the plunge control perturbation also produces little to no change in flutter speed. When control is applied to the pitching movement, the flutter speed is increased with increasing gain magnitude in the negative direction. Figure 16 exhibits the relationship between pitching control gain and flutter speed for the unsteady model, as well as the effects of control perturbation, $\delta_{\alpha}$. For $\delta_{\alpha}$ values less that 0.05 the flutter curve remains constant. After 0.05 the flutter speed will always be approximately the uncontrolled value, in this case $19.38 \mathrm{~m} / \mathrm{s}$. Figure 17 shows the pitch control's influence on the LCO amplitude of the unsteady model. As the control gain is increased in the negative direction the LCO amplitude is decreased. As the control perturbation is increased the LCO amplitude decreases with negative gain. This analysis can provide a guideline for choosing appropriate perturbation-gain combinations to achieve the maximum LCO amplitude performance.

The LCO amplitude of the unsteady model can be suppressed with pitch control. Figure 18 shows the time histories for a system, with a free stream velocity of $U=20 \mathrm{~m} / \mathrm{s}$, which is slightly above flutter speed. With a control gain of -0.25 , the vibration is completely damped. Even before flutter speed, the limiting pitch control has a positive effect on the vibrations. A small control gain in the negative direction makes the LCO amplitude half

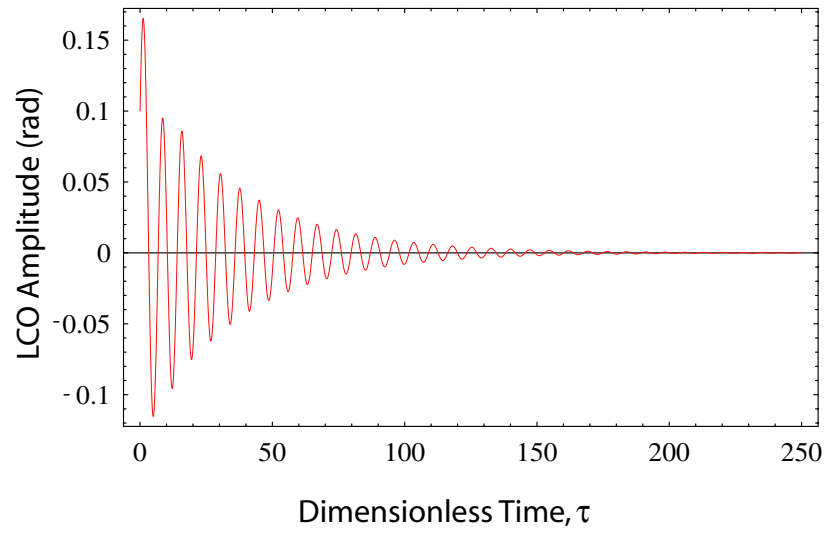

(a) No Pitch Control

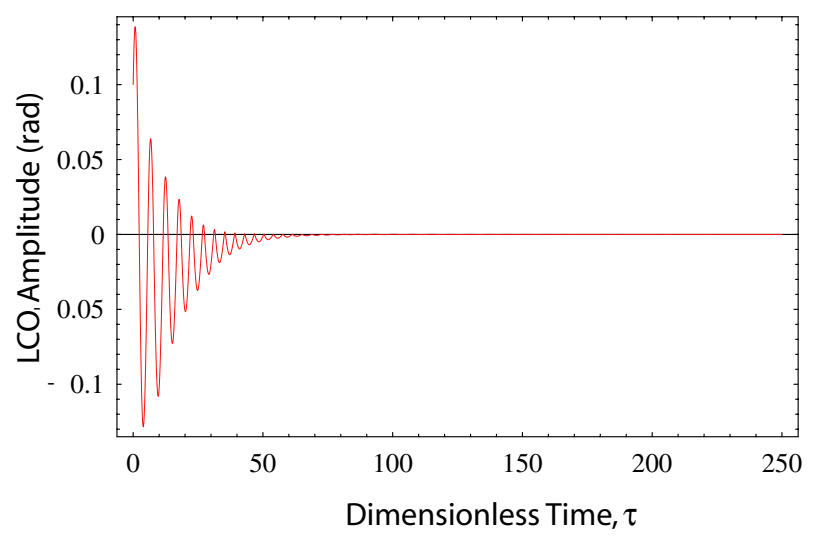

(b) Pitch Control Gain, $g_{\alpha}=-0.25$

Fig. 19. Time histories of unsteady airfoil at $U=17 \mathrm{~m} / \mathrm{s}$ (a) without control, (b) with pitching control gain, $g_{\alpha}=$ $-0.25, \delta_{\alpha}=0.001$, showing that before flutter speed the control decreases the vibrations.

as large. Figure 19 shows the time histories before flutter, with and without control.

\section{Conclusions}

The results presented in this work enhance the scope and reliability of the aeroelastic analysis and design criteria of light and flexible wings. This simple and novel control strategy can supplement or replace conventional control devices, such as flaps and ailerons, with synthetic jet, or morphing type actuators to create a seamless aircraft with no moving control surfaces. For an airfoil in unsteady flow, the applied control was shown to both suppress the LCO, and extend the flutter boundary. The analysis performed in this paper can serve as guideline for selecting appropriate control gains and perturbation values to maximize performance. 
These results contribute, through control methodology, to the avoidance of design and operational pitfalls that may result in catastrophic failures, and can improve ride comfort. Since the power required to implement this design is asymptotically zero, the actuators needed would be relatively simple. The uncomplicated form of our control plant promises affordable testing and later implementation.

\section{Acknowledgments}

Christina Rubillo was supported for this work by the National Science Foundation under DGE0338216. Erik Bollt was supported through NSF under DMS-0404778. Pier Marzocca would like to thank Prof. L. Librescu of Virginia Tech for his support, guidance and collaboration.

\section{Nomenclature}

$$
\begin{aligned}
a= & \text { Dimensionless elastic axis position } \\
& \text { measured from the midchord, posi- } \\
& \text { tive aft } \\
b= & \text { Half-chord length } \\
C_{L \alpha}= & \text { Lift-curve slope } \\
\varepsilon= & \text { Nonlinear stiffness factor } \\
g_{h}, g_{\alpha}= & \text { Plunging and pitching control gain, } \\
& \text { respectively } \\
h, \alpha= & \text { Plunging displacement and the twist } \\
& \text { angle about the pitch axis, respectively } \\
K_{h}, K_{\alpha}= & \text { Spring stiffness in plunge and pitch } \\
& \text { directions, respectively } \\
m= & \text { Airfoil mass per unit span } \\
Q_{c, h} Q_{c, \alpha}= & \text { Plunge and pitch control respectively } \\
Q_{h}, Q_{\alpha}= & \text { Aerodynamic lift and moment, respec- } \\
& \text { tively } \\
r_{\alpha}= & \text { Nondimensional radius of gyration } \\
& \text { about elastic axis (EA) } \\
t, \tau_{0}, \tau= & \text { Time variables and dimensionless time, } \\
& (\equiv t U / b) \\
U= & \text { Free-stream speed } \\
U_{F}= & \text { Flutter Speed } \\
x_{\alpha}= & \text { Nondimensional static unbalance of the } \\
& \text { airfoil about its elastic axis; CG-EA } \\
& \text { offset } \\
\delta_{h}, \delta_{\alpha}= & \text { Plunging and pitching control pertur- } \\
& \text { bation } \\
\zeta_{h}= & \text { Damping coefficients in plunging }=\zeta_{h} / \\
& \left(\pi \rho b^{4} \omega_{\alpha}\right) \\
\zeta_{\alpha}= & \text { Damping coefficients in plunging }=\zeta_{\alpha} / \\
& \left(\pi \rho b^{4} \omega_{\alpha}\right)
\end{aligned}
$$

$\zeta=$ Nondimensional damping coefficient

$\phi(\tau)=$ Wagner indicial function

$\mu=$ airfoil-to-air mass ratio, $m / \pi \rho b^{2}$

$\rho=$ Air density

$\omega_{h}, \omega_{\alpha}=$ Uncoupled natural frequency in bending and torsion, respectively

$\bar{\omega}=$ Frequency ratio, $\omega_{h} / \omega_{\alpha}$

$(\cdot)=\mathrm{d}() / \mathrm{d} \tau$, differentiation with respect to the nondimensional time $\tau$

\section{References}

Barker, J. M. \& Balas, G. J. [2000] "Comparing linear parameter-varying gain-scheduled control techniques for active flutter suppression," J. Guid. Contr. Dyn. 23, 948-955.

Berggren, D. [2004] "Investigation of limit cycle oscillations for a wing section with nonlinear stiffness," Aerosp. Sci. Technol. 8, 27-34.

Bollt, E. [2003] "Targeting control of chaotic systems," in Chaos and Bifurcations Control: Theory and Applications, eds. Chen, G., Yu, X. \& Hill, D. J. (SpringerVerlag), pp. 1-25.

Coller, B. D. \& Chamara, P. A. [2004] "Structural nonlinearities and the nature of the classic flutter instability," J. Sound Vibr. 277, 711-739.

Corron, N. J., Pethel, S. D. \& Hopper, B. A. [2000] "Controlling chaos with simple limiters," Phys. Rev. Lett. 84, 3835-3838.

Corron, N. J. \& Pethel, S. D. [2002] "Control of long periodic orbits and arbitrary trajectories in chaotic systems using dynamic limiting," Chaos 12, 1-7.

Dowell, E. H. [1978] A Modern Course in Aeroelasticity (Sijthoff and Noordhoff, Rockville, MD).

Dowell, E. H., Edwards, J. \& Strganac, T. [2003] "Nonlinear aeroelasticity," J. Aircraft 40, 857-874.

Epureanu, B. I., Tang, L. S. \& Paidoussis, M. P. [2004] "Coherent structures and their influence on dynamics of aeroelastic panels," Int. J. Non-Lin. Mech. 39, 977-991.

Friedmann, P., Guillot, D. \& Presente, E. [1997] "Adaptive control of aeroelastic instabilities in transonic flow and its scaling," J. Guid. Contr. Dyn. 20, 1190-1199.

Fung, Y. C. [1969] An Introduction to the Theory of Aeroelasticity (Dover, NY).

Guillot, D. M. \& Friedmann, P. P. [2000] "Fundamental aeroservoelastic study combining unsteady computational fluid mechanics with adaptive control," $J$. Guid. Cont. Dyn. 23, 1117-1126.

Hodges, D. H. \& Pierce, G. A. [2002] Introduction to Structural Dynamics and Aeroelasticity (Cambridge University Press).

Horikawa, H. \& Dowell, E. H. [1979] "An elementary explanation of the flutter mechanism with active feedback controls," J. Aircraft 16, 225-232. 
Jones, R. T. [1940] "The unsteady lift of a wing of finite aspect ratio," NACA Rept. 681.

Ko, J., Kurdila, A. J. \& Strganac, T. W. [1997] "Nonlinear control of a prototypical wing section with torsional nonlinearity," J. Guid. Contr. Dyn. 20, 1181-1189.

Lee, B. H. K., Price, S. J. \& Wong, Y. S. [1999] "Nonlinear aeroelastic analysis of airfoils: Bifurcation and chaos," Progr. Aerosp. Sci. 35, 205-334.

Librescu, L., Marzocca, P. \& Silva, W. A. [2002] "Post-flutter instability of a shell type structures in hypersonic flow field," J. Spacecraft and Rockets $\mathbf{3 9}$, 802-812.

Librescu, L. Chiocchia, G. \& Marzocca, P. [2003a] "Implications of cubic physical/aerodynamic nonlinearities on the character of the flutter instability boundary," Int. J. Nonlin. Mech. 38, 173-199.

Librescu, L., Na, S., Marzocca, P., Chung, C. \& Kwak, M. K. [2003b] "Active aeroelastic control of 2-D wingflap systems in an incompressible flow field," Proc. 44th AIAA/ASME/ASCE/ASC Structures, Structural Dynamics, and Materials Conference, Norfolk, VA 7-10 April, 2003. AIAA Paper 2003-1414.

Librescu, L. \& Marzocca, P. [2005] "Advances in the linear/nonlinear control of aeroelastic structural systems," Acta Mechanica, DOI 10.1007/s00707-0050222-6.

Lind, R. \& Brenner, M. [1999] Robust Aeroservoelastic Analysis (Springer-Verlag, UK).

Marzocca, P., Librescu, L. \& Chiocchia, G. [2001] "Aeroelastic response of 2-D lifting surfaces to gust and arbitrary explosive loading signatures," Int. J. Impact Engin. 25, 41-65.

Marzocca, P., Librescu, L. \& Silva, W. A. [2002a] "Aeroelastic response of nonlinear wing section by functional series technique," AIAA J. 40, 813-824.

Marzocca, P., Librescu, L. \& Silva, W. A. [2002b] "Flutter, post-flutter and control of a supersonic 2-D lifting surface," J. Guid. Cont. Dyn. 25, 962-970.

Mukhopadhyay, V. [2000] "Benchmark active control technology," J. Guid. Contr. Dyn. Part-I 23, 913-960; Part-II 23, 1093-1139; [2001] Part-III 24, 146-192.
Mukhopadhyay, V. [2003] "Historical perspective on analysis and control of aeroelastic responses," J. Guid. Contr. Dyn. 26, 673-684.

Myneni, K., Barr, T. A., Corron, N. J. \& Pethel, S. D. [1999] "New method for the control of fast chaotic oscillations," Phys. Rev. Lett. 83, 2175-2178.

Qin, Z., Marzocca, P. \& Librescu, L. [2002] "Aeroelastic instability and response of advanced aircraft wings at subsonic flight speeds," Aerosp. Sci. Technol. 6, 195-208.

Scanlan, R. H. \& Rosenbaum, R. [1951] Introduction to the Study of Aircraft Vibration and Flutter (Macmillan Company, NY).

Scott, R. C. \& Pado, L. E. [2000] "Active control of wind-tunnel model aeroelastic response using neural networks," J. Guid. Cont. Dyn. 2, 1100-1108.

Shahrzad, P. \& Mahzoon, M. [2002] "Limit cycle flutter of airfoils in steady and unsteady flows," J. Sound Vibr. 256, 213-225.

Toumit, S. \& Darracq, D. [2000] "Simulation of flutter boundary and Hopf bifurcation of a 2DOF airfoil," Proc. European Congress on Computational Methods in Applied Sciences and Engineering (ECCOMAS). Barcelona, Spain 11-14, September, 2000.

Vipperman, J. S., Clark, R. L., Conner, M. D. \& Dowell, E. H. [1998] "Investigation of the experimental active control of a typical section airfoil using trailing edge flap," J. Aircraft 35, 224-229.

Wolfram, S. [1999] The Mathematica Book, 4th edition (Wolfram Media, Champaign, IL).

Xing, W. \& Singh, S. N. [2000] "Adaptive output feedback control of a nonlinear aeroelastic structure," J. Guid. Contr. Dyn. 23, 1109-1116.

Zeng, Y. \& Singh, S. N. [1998] "Output feedback variable structure adaptive control of aeroelastic systems," J. Guid. Contr. Dyn. 21, 830-837.

Zhang, R. \& Singh, S. N. [2001] "Adaptive output feedback control of an aeroelastic system with unstructured uncertainties," J. Guid. Contr. Dyn. 24, 502-509. 